\title{
COMMUNICATIONS UNCERTAINTIES IN ISOLATED MULTI-MICROGRID CONTROL SYSTEMS
}

\author{
D. Rua \\ INESC Porto, FEUP \\ Porto, Portugal \\ drua@inescporto.pt
}

\author{
J. A. Peças Lopes \\ INESC Porto, FEUP \\ Porto, Portugal \\ jpl@fe.up.pt
}

\author{
J. Ruela \\ INESC Porto, FEUP \\ Porto, Portugal \\ jruela@inescporto.pt
}

\begin{abstract}
Microgrids have been explored as active cells capable of providing enhanced control schemes towards a more secure, reliable and efficient operation of $L V$ distribution networks or MV networks when aggregated in a multi-microgrid. These structures, within the smart grid concept, rely on a communications infrastructure that introduces uncertainty in the data exchange process. In this paper such uncertainty is evaluated considering a multi-microgrid operating in isolated mode.
\end{abstract}

Keywords- microgrids; frequency control; communications; uncertainty

\section{INTRODUCTION}

The use of active LV cells, like microgrids (MG), in distribution networks is widely recognized by the research community as a desirable enhanced control mechanism to provide secure and reliable power distribution to the different participants of a distribution grid [1]-[3]. MGs and multimicrogrids (MMGs) control strategies are aligned with the smart grid (SG) concept where different control schemes supported by a bidirectional communications infrastructure are able to promote the participation of different entities, namely distributed energy resources (DER) [4]-[5]. However, the use of communications systems introduces a degree of uncertainty that should be considered when designing and evaluating control schemes like those associated with such hierarchical systems. Local and centralized control strategies are involved and, as such, it is important to characterize how the communications systems can support the necessary control strategy to different disturbances that are likely to occur in distribution networks.

The increasing introduction of controllable Distributed Energy Resources (DER) in electrical distribution networks can be regarded as an advantage. A supporting hierarchical control structure that combines central and local control strategies is necessary to ensure the efficient, reliable and secure system operation [6]-[7]. However, bidirectional communications systems with different characteristics need to be considered along with different controllable portfolio composition to provide realistic control services to the system operator.

Paper submitted to Power Systems Computation Conference, August 18-22, 2014, Wroclaw, Poland, organized by Power Systems Computation Conference and Wroclaw University of Technology.
Therefore a hierarchical control scheme that combines central and local control is explored in this paper, namely the adaptation of the control system for the emergency conditions. The variability of the characteristics of communications systems is tested through the use of a Monte Carlo simulation platform that evaluates the system responses based on probabilistic data delays and information losses.

\section{CONTROL AND COMMUNICATIONS}

The control schemes envisaged for the smart grids require a communications infrastructure to support different types of applications [8]-[9]. However there is a degree of uncertainty associated with the performance of the communications systems that can introduce variable delays or even cause the loss of information. These phenomena can have a significant impact on control strategies, especially in electric networks with limited controllable resources capable of handling disturbances. The impact is higher when the electric system is operating in emergency or near emergency state. This is aggravated when an islanding process occurs, isolating part of the grid, which can jeopardize the network survival capability. It is precisely under these conditions that communications need to be evaluated since the operating conditions are very stringent and the combined action of local and centralized control strategies play a crucial role.

\section{A. Control Strategy}

A MMG control scheme based on a hierarchical control approach defined and described in detail in [10], using the same mathematical models, was adopted in this research. A discrete frequency control scheme for islanded operation that includes a periodic set-point exchange mechanism is proposed by the authors. The set-points are always exchanged in a predefined sample time, which can take into account the delay of information, but the proposed scheme has a few limitations when uncertainties associated with communications need to be considered. Hence a reformulation of the control strategy was performed to allow the evaluation of different operating conditions using a variable sample time approach, which means that set-points can be issued at different time intervals allowing the control scheme to adapt to different conditions.

Hence, variable control time steps can be defined for instance to deal separately with normal and emergency states 
of operation. The control system also allows the use of a variable controllable portfolio with different operating conditions. A local discrete load shedding mechanism is available, which can be configured with a predefined load shed step per load or per area (LV and MV). A communications block was designed to allow the emulation of uncertainties associated with the communications infrastructure.

The proposed frequency control system behaves like an Automatic Generation Control (AGC) system, using a proportional-integral (PI) controller. Its main function is to restore the frequency value when deviations occur, ensuring that the frequency deviation (error) converges to zero in the steady state. To this end the PI controller will output a power variation $(\Delta \mathrm{P})$ according to an input frequency variation $(\Delta \mathrm{f})$ and the internal parameters (gains). This $\Delta \mathrm{P}$ corresponds to an exchange in either generation or load within the islanded MMG. Fig. 1 shows the typical representation of the PI block transfer function, with the respective gains. These are tuned through experimentation and are based on the quality of the output overall response, since the system plant is in most cases complex to model accurately. Moreover, the system is likely to suffer changes of the stochastic behavior of some components and ultimately due to the impact that communications can have on the system response.

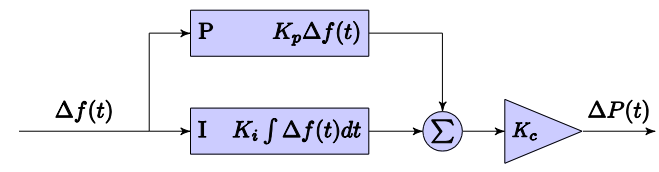

Fig. 1. PI controller transfer function.

The elimination of the frequency deviation is based on $\Delta \mathrm{P}$, but to prevent the extemporaneous action of the hierarchical control a power variation threshold, $\Delta \mathrm{P}_{\text {thrsh }}$, is defined. This prevents the central control from continuously varying the power of the available controllable portfolio in the MMG. It also allows the primary control to take over in between.

The set-points exchanged inside the MMG follow an optimization scheme that finds the best solution from the available controllable devices for each $\Delta \mathrm{P}$ value computed at each control time step. Hence a Mixed Integer Linear Programing (MILP) formulation was adopted, representing a difficult problem to be solved. The MILP mathematical formulation is presented in Eq.1. This is a convenient alternative to a merit-order approach since it allows the inclusion of restrictions more easily. For each control time step a MILP problem needs to be solved, which means that the bounds of each variable need to be update in each time step.

$\min Z=\sum_{i=1}^{m} c_{i} x_{i}+\sum_{j=1}^{n} d_{j} \Delta P_{s_{j}} y_{j}$, with $\Delta P_{s_{j}}=\frac{\Delta P_{\text {Total }_{j}}}{s_{j}}$

subject to:

$$
\begin{gathered}
\sum_{i=1}^{m} x_{i}+\sum_{j=1}^{n} \Delta P_{s_{j}} y_{j}=\min \left(\left|\Delta P_{\text {Total }}\right| ;\left|\sum_{i=1}^{m} x_{i}+\sum_{j=1}^{n} \Delta P_{s_{j}} y_{j}\right|\right) \\
\left|\Delta P_{\text {Total }}\right|>\Delta P_{\text {thrsh }}
\end{gathered}
$$

$$
\begin{aligned}
& l b_{i} \leq x_{i} \leq u b_{i}, \text { with } x_{i} \in \mathbb{R} \\
& l b_{j} \leq y_{j} \leq u b_{j}, \text { with } y_{j} \in \mathbb{N}
\end{aligned}
$$

where:

$c_{i}$ : cost per unit of power of generator $i$

$x_{i}$ : amount of varied power assigned to generator $i$

$m$ : total number of controllable generators

$l b_{i}$ : allowable lower bound of power variation of generator $i$

$u b_{i}$ : allowable upper bound of power variation of generator $i$

$d_{j}$ : cost per unit of power of load $j$

$y_{j}$ : number of varied steps of load $j$

$n$ : total number of controllable loads

$l b_{j}$ : allowable lower bound of step variation of load $j$

$u b_{j}$ : allowable upper bound of step variation of load $j$

$s_{j}$ : total number of steps of load $j$

\section{B. Control Architecture}

The control scheme is based on a set-point exchange mechanism associated with the hierarchical structure of a multi-microgrid. The set-point exchange is illustrated in Fig.2 where two levels are defined.

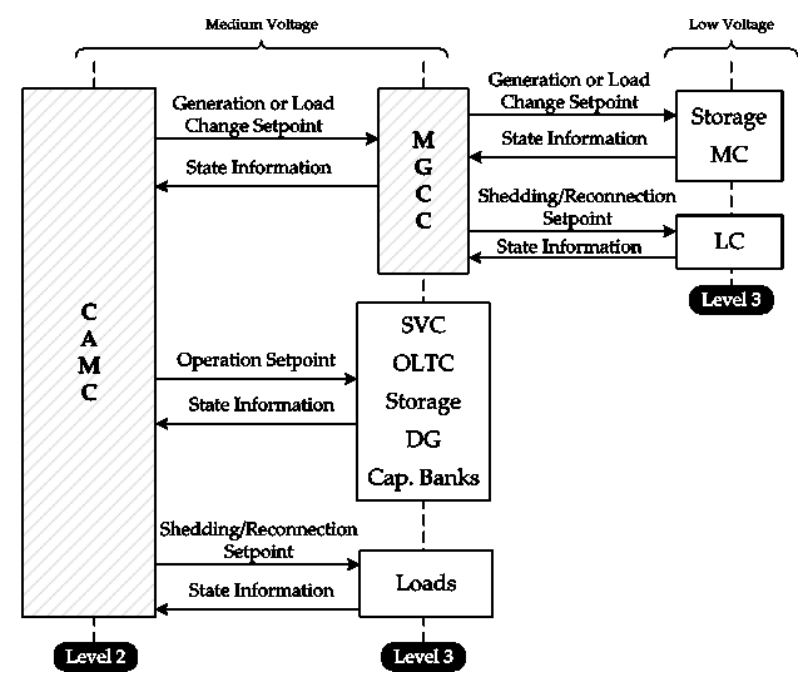

Fig. 2. MMG information exchange.

The Central Autonomous Management Controller (CAMC) is responsible for managing and controlling the portfolio of DER at the MV level, which includes large generators, loads and microgrids [11]. Conversely, the Microgrid Central Controller (MGCC) is responsible for the MG controllable devices. The CAMC is not aware of the specific composition of each MG but knows which one of them is controllable and their respective operational limits. This means that the optimization scheme described earlier has to run globally at the MV level and then run at each LV microgrid by the MGCC in order to find the necessary set-points to be exchanged at each level.

\section{Uncertainty of Communications}

The impact that communications can have on the information exchange process underlying the control scheme described earlier needs to be evaluated considering globally 
phenomena like delays and losses. The delay uncertainty is used as an aggregate metric that can be regarded as the sum of several components like:

- Propagation time in the communications medium;

- Processing time in nodes, which includes protocol conversions/transitions;

- Retransmissions due to failures;

- Transmission time in multi-hop communications structures (store and forward).

Similarly, losses can also aggregate different types of events that will result in the loss of information, such as:

- Loss due to adverse propagation conditions or impairments in the communications channel (recovery strategies can be used but losses may be permanent or still occur after a number of retransmissions);

- Information at the receiving end was correctly interpreted but is not valid.

In order to evaluate the impact of communications an emulation module was added to the set-point exchange mechanism. A probabilistic process was associated with the control scheme in order to introduce realism due to the uncertainties in the data exchange.

Despite the fact that different strategies can be employed to deal with delays and losses, namely recovery schemes, it was considered that losses, when occurring, are permanent and no retransmissions are triggered. This creates an unfavorable scenario for the control scheme to handle.

The integration of the delay variation in the control scheme was introduced using a random number generator based on a normal distribution according to previously specified mean and standard deviation values. The normal distribution is defined by the well-known probability density function:

$$
f(x)=\frac{1}{\sigma \sqrt{2 \pi}} e^{-\frac{(x-\mu)^{2}}{2 \sigma^{2}}} \text { for } \sigma>0 \text { and }-\infty<\mu<+\infty
$$

The losses in the data exchanged between the control management entities were introduced through a drawing mechanism; after knowing the number of targets to which setpoints have to be sent within the control action a finite number of failures was randomly generated and indiscriminately assigned to each of these targets. This mechanism is based on the binomial distribution, which in turn is based on a Bernoulli process where a sequence of independent and identical Bernoulli trials is considered. Bernoulli trials are referred to as random experiments where only a binary result is possible: success or failure. The binomial distribution is defined by the probability density function:

$$
f(k ; n, p)=\left\{\begin{array}{cl}
\left(\begin{array}{l}
n \\
k
\end{array}\right) p^{k}(1-p)^{n-k}, & \text { where } k=[0,1, \ldots n] \\
0 & , \text { otherwise }
\end{array}\right.
$$

Hence the loss of information is emulated through a random number generator that, based on a binomial distribution, is able to generate $k$ out of possible $n$ failures with a determined probability of error $p$. Similarly to delay, losses at the MV level will have a direct impact at subsequent LV MGs, which means that the affected active cells will have no control set-points issued.

\section{THE TEST SYSTEM}

A test MV network with distributed generation units and controllable loads was implemented in Eurostag, which is interconnected to an external control MMG structure implemented in Python. This network is composed of two different zones, one rural and the other urban, with different characteristics. The rural part is constituted of long overhead lines with significant voltage losses, whereas the urban part has shorter and underground electric cables. In this test network there are active MGs, distributed generation (diesel, CHP and wind turbines), storage devices, and $\mathrm{MV}$ and $\mathrm{LV}$ responsive loads. The control algorithm has a predefined sample time over which it is able to collect state information from the MMG and issue control set-points. Fig.3 depicts the test network and its components.

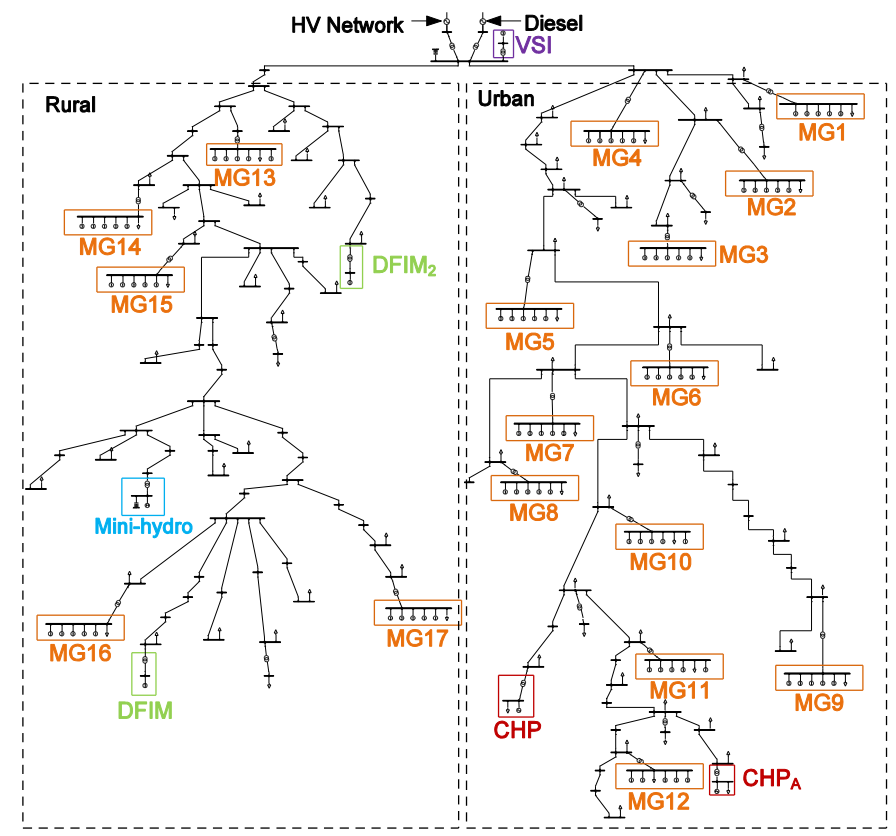

Fig. 3. Test network.

Each MG is composed of a generation portfolio, which may include photovoltaic, micro wind turbines, gas microturbines and fuel cells. Controllable loads as well as storage devices controlled and interfaced by means of Voltage Source Inverters (VSI) can also be part of MGs. Only gas microturbines and controllable loads are set to participate in the secondary frequency control. From the available MGs only six of them are controllable, thus allowing a scenario of limited controllability to be considered.

In terms of secondary control, a frequency deadband and a threshold for power variation are defined to prevent extemporaneous control actions thus inhibiting the response to small frequency variations detected by the PI controller. This allows the primary control and electromechanical transients to 
stabilize before a new round of set-points is issued. Hence a frequency deadband of $1 \%$ was established along with a minimum of $20 \mathrm{~kW}$ of $\Delta \mathrm{P}$ to trigger a central control action.

\section{RESULTS AND DISCUSSION}

An isolation event was created in the MV distribution grid to allow the evaluation of the control system over a communications infrastructure subjected to uncertainties. The MV MMG is isolated due to an upstream disturbance and prior to this event is assumed to be in a steady state and drawing 4.83 MW from the upstream network. The generation available inside the MMG totals 4.4 MVA, meaning that there is a shortage of power supply that has to be compensated through a centralized load shedding procedure. The isolation occurs at $\mathrm{t}=25 \mathrm{~s}$ and the MMG control scheme is only aware of the disturbance when a new system observation is performed, which may take up to $t_{s}$ seconds. This value includes also the time associated with the execution of the control algorithm.

The system response to the isolation event is depicted in Fig.4 where it is possible to see the outcome when no secondary control action is taken, with the inhibition of the MMG control.

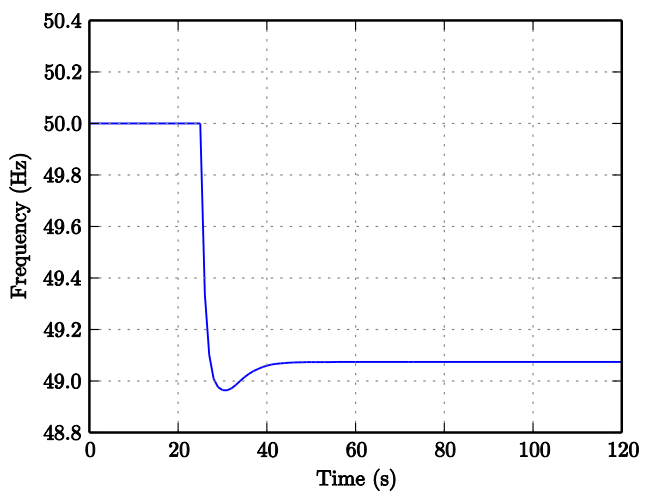

Fig. 4. System response without secondary control.

The centralized MMG control system was set to respond to operating conditions by issuing set-points. A normal control sample time $t_{s}=5 \mathrm{~s}$ is assumed when operating under normal conditions; an emergency time sample variation $t_{e}$ was explored to understand the benefits in terms of system response of having the control system reacting at different sample times, as depicted in Fig.5.

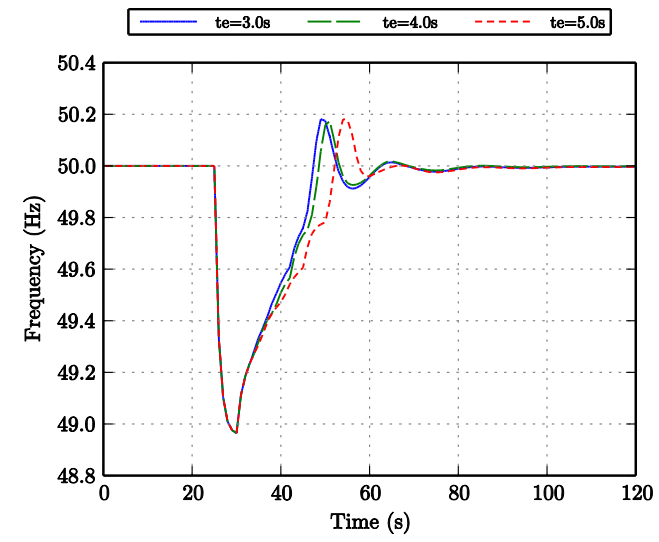

Fig. 5. System response for different $t_{e}$ values.
The $t_{s}$ value is the same for all cases, which means that the initial response is exactly the same, as expected. This assumes that the disturbance detection is kept constant. As expected, for lower $t_{e}$ values the control scheme is able to react earlier, thus the differences in the system response. However, in steady state, only little and generally negligible differences are detectable. This suggests that narrowing the time between observations does not allow significant benefits in terms of frequency response. Furthermore, lower $t_{e}$ values were shown to trigger a larger number of set-points that need to be sent by each controller. This can have consequences on the mechanical stress induced by the increase in the number of power variation set-points issued to electric generators, with arguable benefits for the overall frequency response.

An assessment of the impact of delays was conducted, by introducing a random delay and jitter to each set-point exchange at the different levels, MV and LV, of the control scheme. This includes the system observation and the time that a set-point takes to be transmitted, processed and implemented. Fig.6 illustrates the different frequency responses when different average delay values are considered. Since no particular advantages were deemed from small $t_{e}$ value a default $4 \mathrm{~s}$ sample time was assumed for the emergency operation.

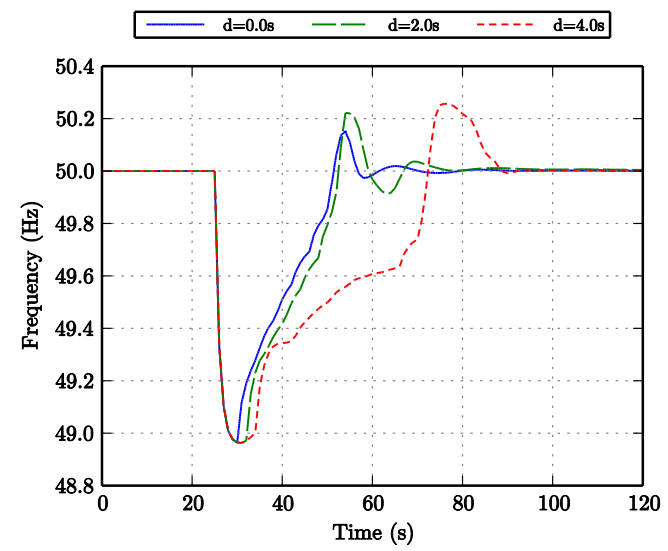

Fig. 6. System response in the presence of delays.

An ideal response with no delay, $\mathrm{d}=0 \mathrm{~s}$, corresponds to an ideal case similar to the one presented earlier when demonstrating the impact of the secondary control. For $\mathrm{d}=2 \mathrm{~s}$ it is clear the deviation from the ideal response namely in terms of increased intermediate oscillations but the nominal frequency value is fully restored. Nonetheless, for the case where $\mathrm{d}=4 \mathrm{~s}$, the deviation is quite significant with a substantially delayed response and a slower convergence towards the steady state. An average delay with the same magnitude as the default $t_{e}=4 \mathrm{~s}$ means that some set-points are issued before the previous round is completed. This effect is aggravated by the fact that delays are cumulative between levels, meaning that most set-points within a LV MG are received with a delay well beyond the MMG $t_{e}$ emergency sample time. Under these conditions, the centralized control scheme issues set-points that can negatively affect the system response since new set-points can be dispatched in a counterproductive fashion. Furthermore, in this particular case, a higher number of set-points was required towards the 
frequency restoration after the disturbance, since the control system often issued control set-points that end up to be outdated.

Part of the discrete load shedding mechanism is illustrated in Fig.7 considering the same delay values presented before. One of the consequences of high delay values is a higher load shedding.
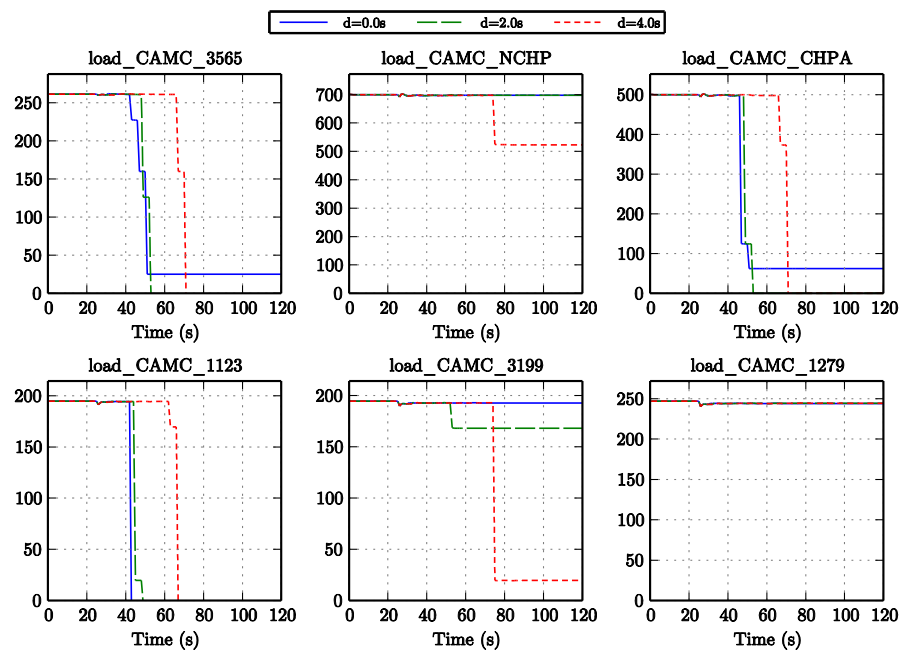

Fig. 7. MV load shedding.

Similarly, Fig. 8 depicts the power variation of the centrally controlled MV CHP units as a consequence of the exchanged control set-points. Once more the presence of delays and the discrete nature of the load shedding process are visible in the intermediate corrections that these generators are requested to fulfill.
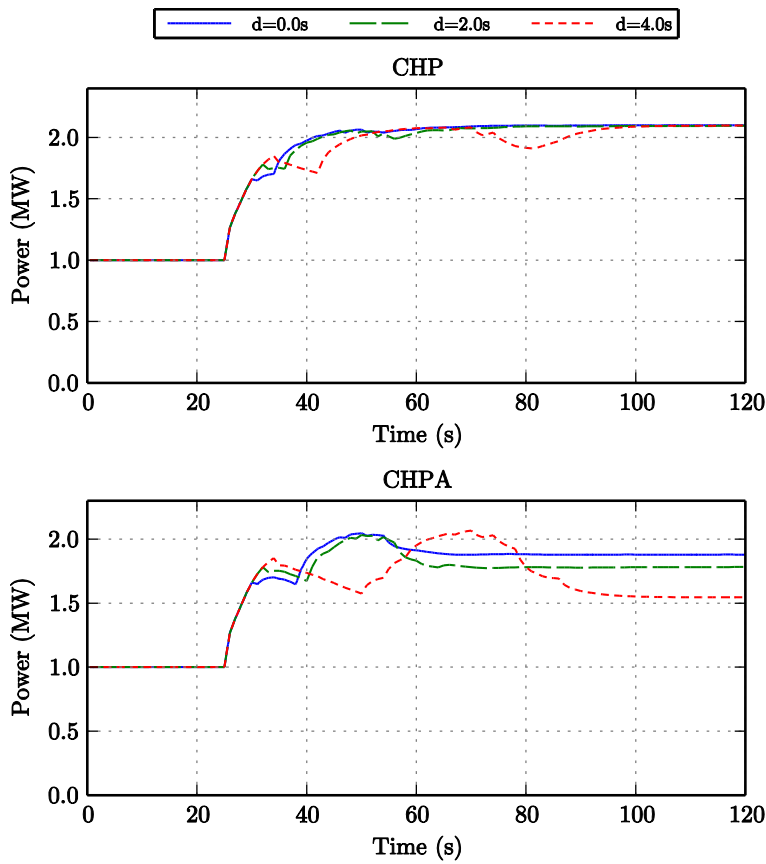

Fig. 8. MV controllable generation.
The response of one of the controllable MGs is portrayed in Fig.9. It is possible to see the response of the microturbines and the load shedding, which are both part of the secondary control, as well as the primary control response of the associated VSI. For the latter it is possible to see that for $d=4 \mathrm{~s}$ the inverter is requested to have a longer contribution in supporting the frequency restoration procedure.

An evaluation of the loss of information associated with the control set-point exchange procedure was carried out. To that end a set of predefined loss ratio values was established for all set-points exchanged within the MMG system. This means that each set-point exchange has the same probability of being lost according to the previously mentioned predefined loss ratio. No retransmissions are considered due to the counterproductive effect of exchanging outdated set-points as it so happened when delays were excessive.

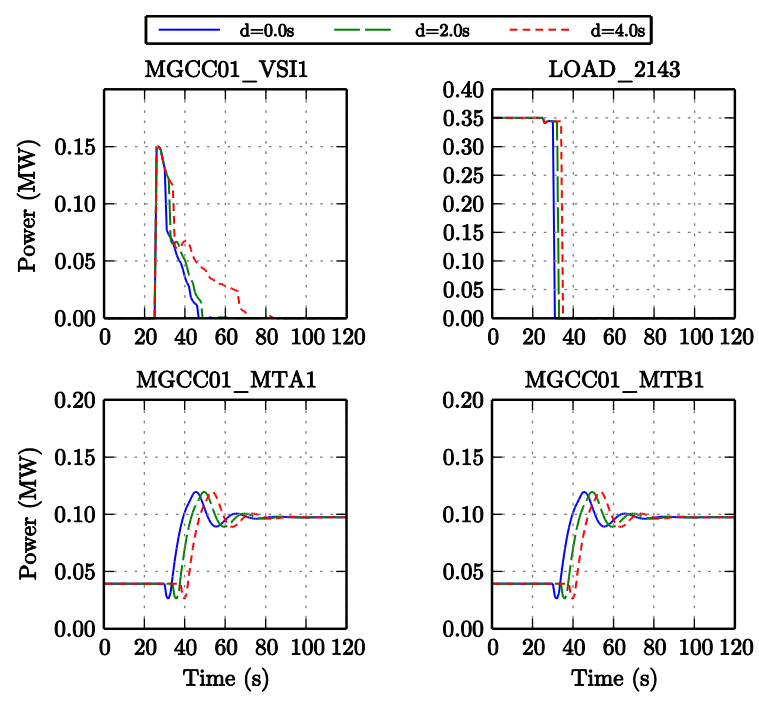

Fig. 9. Controllable MG response.

A Monte Carlo simulation platform was implemented as a convenient way of evaluating the impact of losses at different targets and at different time intervals of the control scheme. A set of 1000 simulation runs was carried out for different target loss ratios and the outcome is presented in Fig. 10.

For each of the loss ratio values an ideal response, corresponding to no losses, is presented in the black dashed line. The average system frequency response is presented in green, providing an overall perspective of the impact of delays in the control scheme. The standard and maximum upper and lower deviations allow the perception of the dispersion from the average and extreme deviation, respectively.

In all cases, the average curves show a delayed response when compared to the ideal case. Another visible aspect is the fact that the ideal case bounds the upper deviations from the average until the overshoot is reached, making it in fact the fastest response. The maximum deviations show the system response when higher unbalances between the power generation and load shedding procedure occur. 


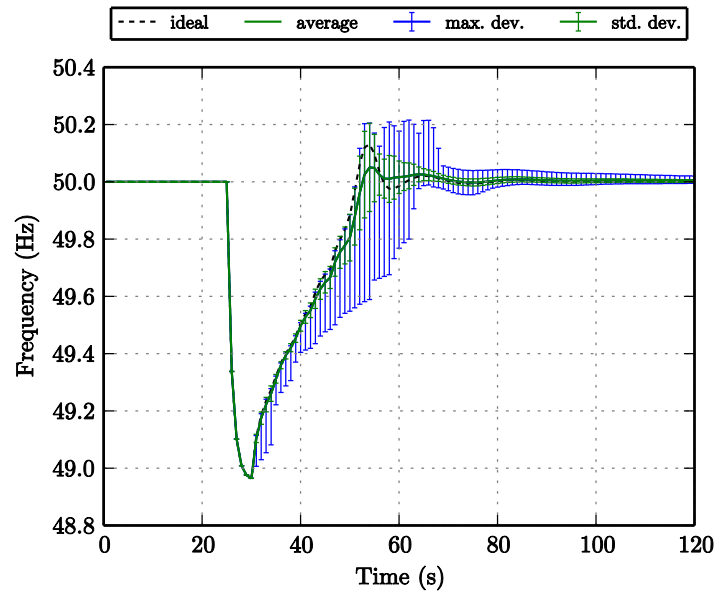

(a) Loss ratio at $5 \%$

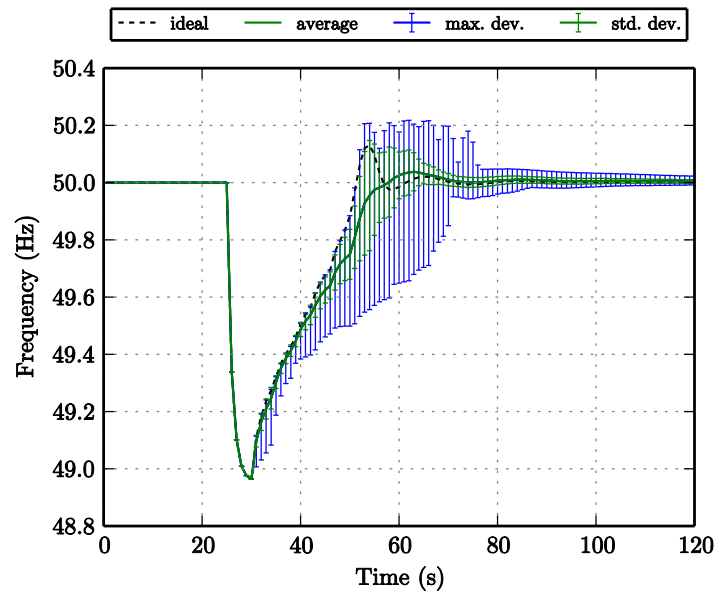

(b) Loss ratio at $10 \%$

Fig. 10. Impact of losses in the system response.

In Fig. 11 the maximum frequency deviation values of the system response, when compared to the ideal response, are presented. The frequency deviation values are plotted as a function of the loss ratio of each run of the Monte Carlo simulation considering the two predefined loss ratio targets $5 \%$ and $10 \%$. It is noticeable that higher loss ratio values tend to produce larger frequency deviations, as expected. However for the same value of data loss ratio different maximum frequency deviation values are achieved.

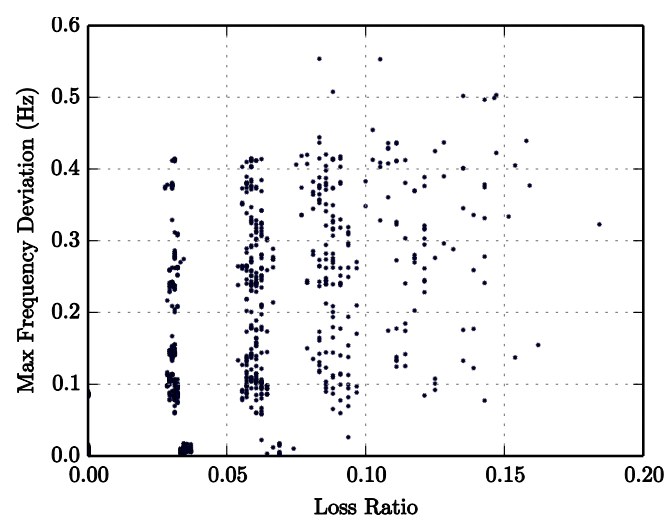

(a) For a target loss ratio of $5 \%$

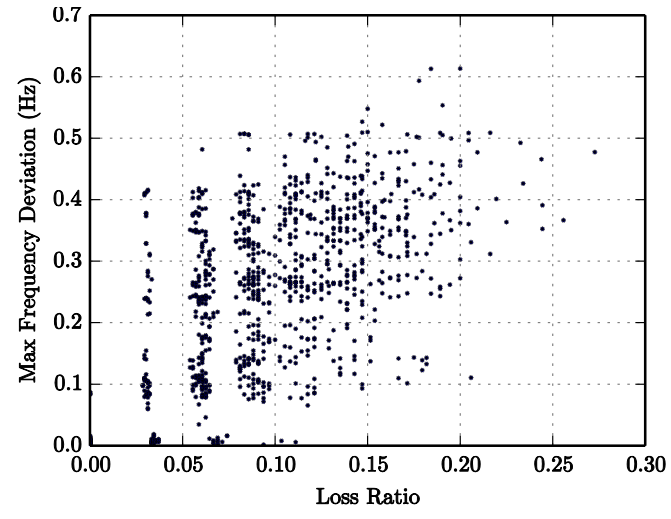

(b) For a target loss ratio of $10 \%$

Fig. 11. Loss ratio per simulation run.

One reason associated with this behavior is related with the fact that although the loss ratio is the same different set-points to different targets were affected. Another reason is the fact that a failure on the same set-point can have different impact depending on the time of occurrence.

The loss ratio for each simulation run of the Monte Carlo implementation is presented in Fig.12.

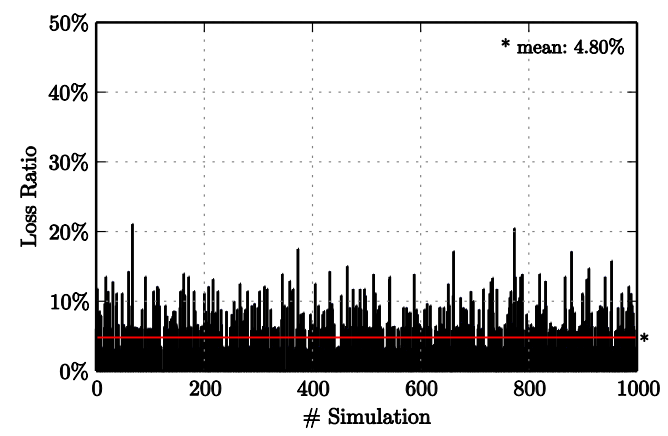

(a) For a target loss ratio of $5 \%$

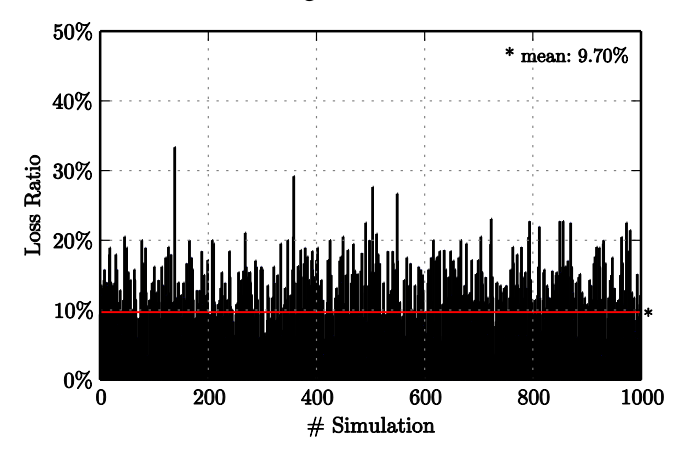

(b) For a target loss ratio of $10 \%$

Fig. 12. Loss ratio per simulation run.

The horizontal line in full represents the average value for each set of simulation for each predefined loss ratio value. In general the values are coherent with loss ratio values established at the beginning of each Monte Carlo run. On one hand it should be noted that a limited number of set-points is exchanged in each control time step, which can introduce significant variations in each run. On the other hand this visible variability of the loss ratio in each simulation run allowed the 
necessary variation in terms of frequency response in order to evaluate the effectiveness of the control scheme when in the presence of information loss uncertainty.

\section{CONCLUSIONS}

The control scheme and test network allowed the assessment of the impact of uncertainties introduced by communications systems in hierarchical structures such as a MMG system. A dynamic and configurable control module capable of interacting with the Eurostag simulation was presented allowing a fast simulation of different cases using PI controller associated with the secondary control, which defined the necessary control set-points to be exchanged within the MMG structure according to the result of a MILP formulation. Smaller sample periods for the emergency operation showed marginal improvements at the expense of a higher number of set-points to be exchanged. The introduction of variable delays in the set-point transmission allowed a non-synchronized implementation of the control actions with negligible impacts on the overall system response, if they are kept below the emergency time sample value. Otherwise the system response varies significantly with outdated set-points being implemented at the same time that newer ones were calculated and dispatched. This created counterproductive actions from the centralized control scheme. The use of different loss ratio values showed that the loss of information can have a significant impact on the system response. Nevertheless, the combination of secondary and primary control actions allowed the system to recover its nominal frequency despite the uncertainty.

\section{ACKNOWLEDGMENT}

The authors would like to thank the support from the ERDF - European Regional Development Fund through the COMPETE Programme (operational programme for competitiveness) and FCT - Fundação para a Ciência e a Tecnologia (Portuguese Foundation for Science and Technology) within projects FCOMP-01-0124-FEDER037281, MicroGrids+EV - FCOMP-01-0124-FEDER-009866, and DYMONDS - CMU-PT/SIA/0043/2009.

\section{REFERENCES}

[1] N. Hatziargyriou et al., "Microgrids - Large Scale Integration of Microgeneration to Low Voltage Grids", CIGRE, 2006.

[2] J. A. Peças. Lopes, C. L. Moreira, and A. G. Madureira. "Defining Control Strategies for MicroGrids Islanded Operation", IEEE Transactions on Power Systems, 21(2):916-924, May 2006.

[3] F. Katiraei, M. R. Iravani, and P. W. Lehn. "Micro-grid Autonomous Operation During and Subsequent to Islanding Process", IEEE Transactions on Power Delivery, 20(1):248-257, Jan.

[4] C. L. Moreira, F. O. Resende, and J. A. Peças Lopes, "Using Low Voltage MicroGrids for Service Restoration", IEEE Transactions on Power Systems, 22(1):395-403, Feb. 2007.

[5] J. Ruela et al., MicroGrids Project Deliverable DF1: Report on Telecommunication Infrastructures and Communication Protocols. Technical report, INESC Porto and LABEIN, 2005.

[6] A. G. Madureira et al., "Advanced control and management functionalities for multi-microgrids", European Transactions on Electrical Power, 21(2):1159-1177, 2011.
[7] F. O. Resende, N. J. Gil, and J. A. Peças Lopes, "Service restoration on distribution systems using Multi-Microgrids", European Transactions on Electrical Power, 21(2):1327-1342, 2011.

[8] M. Shankar, J. Nutaro, J. Stovall, "Evolution of communication and control for electric grid load management", Power and Energy Society General Meeting, 2010 IEEE, pp.1-7, 25-29 July 2010.

[9] Sood, V.K., Fischer, D., Eklund, J.M., Brown, T., "Developing a communication infrastructure for the Smart Grid", Electrical Power \& Energy Conference (EPEC), 2009 IEEE, pp.1-7, 22-23 Oct. 2009.

[10] N. J. Gil, J. A. Peças Lopes; "Hierarchical Frequency Control Scheme for Islanded Multi-Microgrids Operation", Power Tech, 2007 IEEE Lausanne, pp.473-478, 1-5 July 2007.

[11] N. J. Gil, J. A. Peças Lopes, "Exploiting Automated Demand Response, Generation And Storage Capabilities For Hierarchical Frequency Control In Islanded Multi-Microgrids", Proceedings of PSCC2008, 16th Power System Computation Conference, Glasgow, Scotland, August 2008. 
(C) 2014 IEEE. Personal use of this material is permitted. Permission from IEEE must be obtained for all other uses, in any current or future media, including reprinting/republishing this material for advertising or promotional purposes, creating new collective works, for resale or redistribution to servers or lists, or reuse of any copyrighted component of this work in other works. 